\title{
Assistive Technology and Performance Behaviours in Music Improvisation
}

\author{
Ben Challis and Rob Smith \\ Cardiff School of Creative and Cultural Industries, ATRiuM, \\ University of Glamorgan, 86-88 Adam Street, Cardiff, CF24 2FN, UK \\ \{bchallis, rksmith\}@glam.ac.uk
}

\begin{abstract}
The findings from three trial workshops with a group of music-learners with physical disabilities have culminated in an initial design for a novel interactive music-generation system. Using a variety of commercially available musictechnologies in a synchronised set-up, the target group identified those aspects of both music production and accessible interaction that were most appealing and productive. The proposed design places equal emphasis on improvisation and accessibility, generating rhythmic, harmonic and melodic patterns that an individual can trigger and manipulate. The system will also allow a group of improvisers to work together offering variable levels of synchronization based on individual need or preference. A prototype system is currently under development.
\end{abstract}

Keywords: Accessibility, disability, music, improvisation, technology

\section{Introduction}

Though there have been a number of research projects exploring the design of new and novel musical instruments and music systems within a context of accessibility, there has been little focus within these on the relationship between assistive technology and self-expression through improvisation. The work being presented here aims to contribute to the beginning of an on-going dialogue on the concepts and challenges that are likely to arise when the relationship between performer, instrument and creativity is considered against a backdrop of individual needs. By observing and presenting music workshops to a group of adults with a variety of physical challenges, a small research team from the University of Glamorgan, has been able to document a range of issues and opportunities relating to the practical application of a number of potentially quite accessible electronic musical instruments. The overall aim of the project is to design one or more new musical 'tools' that will build upon the best of these opportunities whilst aiming to lessen the impact of the more profound challenges that have been acknowledged along the way.

There are two phases to the project and the first of these is now complete. This has been a process of close observation and analysis with the aim of identifying the key 
challenges for enabling improvised musical activities. The second phase is one of proposing and testing alternative or assistive means for achieving these same creative outcomes. The key researchers are active performers with a shared interest in the use of novel technologies in improvised music. Smith is an experienced practitioner and tutor in Community Music with a research background in free-improvisation and Challis has a background in human-computer interaction and the design of novel technologies for music performance. Although this article represents the completion of the first phase of the overall project, the second phase is in progress and aspects of the design 'brief' are included alongside the closing conclusions.

\section{Improvisation, novel technology and community music}

It is vital, when contemplating using a potentially liberating new musical tool, or devising and testing a novel approach to an existing instrument, to consider how and to what ends the tool might be utilised in the context envisaged. Of course, outcomes and goals frequently predate, shape and guide the creation of tools, but once the tool is in use new avenues and possibilities can be discovered. Some of these new possibilities may be discovered by accident, by a process of play and, in some cases, by wilful abuse or subversion of the tools themselves. All these three areas of activity (celebrating and working with accidents, play, subversion of rules) can easily be viewed as sitting comfortably within the area of activity widely known as free improvisation.

If improvisation is the creation of musical utterances in the moment (often, in the case of experienced and dedicated improvisers, in the moment of performance), and this activity can encompass different genres (folk, Indian classical music) and rule based improvisational systems (bebop) as well as more abstract forms including free improvisation, then we would appear to have in free improvisation a powerfully liberating area of musical practice. Of course reality is more fluid than this. Improvisers can create music of great excitement and beauty entirely from within a genre or set of rules, seemingly working in a mind-space oblivious to any such constraints, or indeed from instinctive syntheses of rule-breaking and rule-keeping strategies: indeed many stable, freely improvising groups (AMM, Supersilent, The Necks) seem to mutually discuss and delineate their own rules or at least areas of interest for exploration when playing.

However, the nature of free improvisation is notoriously difficult to define. Bailey [1] comes down approximately defining free improvisation being free of genre and rules. Others prefer to avoid definitions and contribute to the on-going discussion of improvisation and its uses by action. Stevens [2] divides his work broadly into rhythm and improvisation. Rhythm, in Stevens' methodology (as propounded in Search and Reflect but also in his performances and recordings with groups such as the Spontaneous Music Ensemble) is necessary for group cohesion and finding one's place in a dynamic group statement without destroying that dynamic or the group's coherence. In his introduction to the Improvisation section of Search and Reflect, Stevens tells us that he is building on the preceding rhythm work in dealing with 
'specific processes and skills which help to prepare the way for a sensitive, concentrated approach to creative group interaction and individual spontaneity' [2].

The fact that Stevens' 'Music Workshop Handbook' is widely used throughout the international improvised music communities is testament to the power and potential of this model. Stevens and Search and Reflect were both frequently cited as both starting points and a continuing resource during a previous research project into Free Improvisation Pedagogy in the UK and Norway [3].

Improvisation is also a potentially powerful methodology when trying to overcome the problems that people with special needs have in accessing music-making. It is an important tool in the work of music therapists and related disciplines (such as running music-making sessions with people with physical and/or learning disabilities). Music therapists often need to elicit musical expressions from their clients and make open improvised responses to these utterances and establish a (albeit non-semantic) dialogue with the patient whilst clinically assessing the patients' responses to the therapy. An attitude of watchful patience and the responsive skills of the improviser are key here.

In working with novel technologies in a community or special needs context [4], [5] we have deployed similar improvisation skills as those used in free improvisation performance and musical therapy and related quasi-therapeutic contexts. It is particularly true for instruments where small physical movements can make big dynamic musical changes, that the feedback loop of action-sound-response requires a long learning period where the interface is tested through improvisation and it is here that the improvising-searching-reflecting axis of skills are particularly useful in demonstrating and allowing new users with varying levels of musical skill and manual dexterity to learn to create their own musical expressions using experimental interfaces. If we are in danger of becoming swept away by enthusiasm for the potential power of improvisation, we must not turn our back on the notion of composition.

Composition is a complex and multi-faceted task in itself. At one level however (and this is especially true of electro-acoustic composition) it can be the selection of sounds to form a palette of sounds which can be shaped, manipulated, varied and combined to create a piece or musical performance. Improvisation may be the watchword for creating surprising musical expressions with new-technology but there is a necessary process of careful selection and creation of soundfiles to create the raw materials with which to improvise.

On the other hand, a free musical context, where it can be agreed that there is no right or wrong, and therefore all musical gestures are valid, can free up the user to explore the potentials of a configuration of, maybe, new instruments and interfaces that are perhaps only new to that particular user or group of users. Alongside these new and novel interfaces can be used traditional instruments such as percussion or keyboard instruments which could not be synchronised by an external clock or timecode generator, but could operate happily alongside such a community of interfaces. Indeed, this might serve to tighten-up or reinforce the participants' existing timekeeping skills on acoustic instruments. 


\section{Methodology}

The first phase of the project has centred around three contrasting workshop experiences. To provide the project team with an opportunity to fully appreciate the kind of activities that could be regarded as 'typical', a workshop was arranged with a group of adult music-learners with physical disabilities in the South Wales area; the researchers were accommodated within the group as active observers. The group have been together for a number of years and engage in various musical activities using fairly traditional resources (e.g. hand percussion and electronic keyboards). There is a broad spectrum of individual challenges represented within the group with most of the members experiencing some form of difficulty with upper limb movement and/or dexterity; some of the members are also wheelchair users.

Based on the observations from this preliminary workshop, a series of two followup workshops was proposed that would allow the group to work in a different way using a selection of accessible technologies. The first of these workshops was focused on training the group to use the technology (some of which was relatively unfamiliar) and the second workshop was focused on bringing different members of the group together into a number of short improvised music sessions.

\section{Performance Behaviours and Ownership}

This study is ultimately concerned with the design of new 'instruments' to assist with or enable improvised performance. With this in mind, it is of considerable interest as to how assistance can be introduced that does not erode the performer's feeling of musical-involvement and control. Healey [6] refers to this as 'ownership' and stresses that within a context of community music and new technologies this concept is particularly important. A given assistive technology might be quite successful in terms of enabling an individual to produce musical fragments whilst perhaps being relatively disabling in terms of the breadth of originality, contrast, creativity etc. that is on offer. In some ways relating to this, Malloch et al. [7] have proposed a model for referencing and potentially 'placing' some of the limitations and constraints that a digital musical instrument might contain. The model is essentially a continuum that extends between two extremes of performance behaviours. Where the performer is responsible for forming or triggering individual notes, the performance behaviour can be regarded as skill-based. Where the performer has no control over the system beyond starting and stopping playback of a predetermined piece, the performance behaviour can be regarded as model-based. A third performance-behaviour (rulebased) sits partway between these two extremes and encompasses systems and instruments that allow the performer to trigger and perhaps manipulate patterns based on predetermined rule-sets.

This model is a useful tool for helping to define the performance opportunities that any given system might offer, however, it does not appear to immediately consider the nature of systems that are being manipulated by performers with additional individual needs. For example, it could be argued that the skill required by an ablebodied performer to play, for example, a chord shape on a keyboard at a specific time 
is comparable to another performer with physical and dextrous challenges pressing a single (and possibly quite large) switch within a quantised time-scale. With this in mind, the model will be used within discussion to help define the performance behaviours in use but the notion of skill will be kept within a context of individual needs and abilities.

\section{Workshops and observations}

The aim behind the preliminary workshop was one of sharing activities that could be regarded as typical across the group's history. The main musical activity was one of creating group-based rhythms by starting with a single repeating pattern on one or more instruments and then adding further patterns on different instruments. This use of layered rhythms is really quite an effective workshop technique to adopt where there are a large number of players within the group with little or no instrumental training. At a fundamental level, hand-percussion will generally allow even the least experienced of players to contribute something whilst still offering additional complexity through the rhythmic patterns that might be attempted. Percussion instruments also suit the physical needs of many of the group some of whom have quite restricted finger movement such that working with a traditional melodic instrument could be particularly challenging.

Perhaps the most significant observation from the initial workshop these was that although the rhythm-based approach to creating group-music was effective in allowing everyone to contribute, it was also quite restrictive; offering little opportunity to create melodies and/or harmonies. It was also recognised that for quite a number of the group, it would be difficult to engage with many traditional musical instruments that might enable such melodic opportunities. For some of the group, maintaining rhythms on an instrument for any prolonged period could be quite demanding either in terms of physical effort or in terms of maintaining timingaccuracy. However, it was also clear that the various members very much enjoyed functioning as a 'group' musically even though the more members that were involved in any given activity, the harder it became to maintain a sense of rhythmic clarity.

With these key observations in mind, a series of two workshops was proposed to that would allow the group to work with electronic instruments that could suit their specific needs; these would allow them to interact with each other in harmonic and melodic ways as well as rhythmic. Building on the familiarity of working with hand percussion, a Roland Handsonic was the first instrument to be included within this selection. This is a skill-based electronic percussion instrument that offers ten velocity sensitive pads that trigger pre-recorded sounds. Other than offering a wide variety of percussion sounds, the Handsonic also offers melodic instruments (e.g. steel drum) and, though it can sit on a stand or table, it can also be placed quite easily on a performer's lap. In addition to the Handsonic, a Korg Kaossilator Pro was also selected. Primarily aimed at the DJ market, this offers a small touchpad that maps the $\mathrm{XY}$ position of a finger tip onto a specific sound program. Gently touching the pad will trigger either an individual note or a pattern of notes depending on the nature of the chosen sound and some additional settings. Moving the finger around the screen 
will then either move to another note within a predefined scale or alter the nature of the pattern being sounded. At a skill-based level, individual notes can be targeted and triggered but changing to a pattern generating sound quickly allows the performer to interact with rule-based behaviours (e.g. drum rhythms or changing harmonies). The device also allows the results of actions to be sampled as repeating loops such that it could be suggested that it also offers near model-based behaviours. The last instrument to be considered was a synthesiser that offers arpeggiated harmonic patterns. In total, two Handsonics, two Kaossilator Pros and two arpeggiators were used for the workshop. Although the intention for the next workshop would be to have some of these instruments synchronised such that their rhythms and patterns would stay together in tempo, this was not demonstrated at this point. Instead, the function of each instrument was demonstrated and members of the group were then given the opportunity to individually experiment with the various sounds and textures that could be achieved. As opportunities arose, the group was encouraged to use their familiar hand-percussion instruments to build rhythmic accompaniments to the sound being created such that short improvised pieces were achieved within the session.

Comments from the group after individual practice with the various instruments were positive across the board and suggested that all three technologies had something to offer to at least some of the group. Observation of individuals at work with each device appeared to show that the range of sounds and 'textures' on offer was both appealing and engaging and this was confirmed by additional feedback offered by the group. It was suggested that the Handsonic offers access to a broader range of percussion sounds at one sitting than might ordinarily be accessed and that it is particularly responsive and therefore not overly demanding to play. A specific comment was offered on the responsive nature of the Handsonic being easy to reach different volumes with little physical demand adding that "you could modify it [the volume] to suit, I thought it was good". General observation of members of the group using the arpeggiation function of the two synthesisers showed that the generative nature of this function was appealing with the players being able to create and control quite complex harmonic patterns. The Kaossilator Pro proved to be particularly effective for those members of the groups who have very restricted hand and finger movement. One member commented on the nature of the interaction, saying that "it's easy for me to touch the screen, and go along, get difficult beats because, with my hands, I can't do that for long". Another member of the group is a competent keyboard player but still expressed great enthusiasm for the device, commenting that it was particularly easy to interact with and that it produced more complex musical ideas and patterns than might easily be achieved using conventional playing techniques on a traditional keyboard. This same group member offered a comparison between the Handsonic and Kaossilator in terms style of interaction method commenting that "I get tired out with my wrists ... I found that one easier [kaossilator pro], that one [handsonic] was good as well but I think with that one [kaossilator] I could do longer"

The aim with the final workshop was to bring together the same technology that was used previously but within small group-based improvisations. In an attempt to hold together the collective improvisations rhythmically some of the instruments were synchronised using an external MIDI clock. Both Kaossilator Pros and both arpeggiators were synchronised in this way such that whenever a pattern was triggered it would always match the tempo of the others. However, if an instrument 
were to be triggered slightly out of phase in terms of pattern or rhythm it would remain out of phase but still in tempo. So improvisation would be synchronised but not quantised, if a player missed the beat, the pattern would stay off-beat. This would maintain a sense of skill-based behaviour even though the patterns being produce would effectively be rule-based. To help maintain a sense of tonality, each of the Kaossilator Pros was set to work within the key of $\mathrm{C}$ Major. This set of notes corresponds to the white keys on a standard keyboard and is relatively easy for even a novice player to identify. The same was true of the sounds on the Handsonic if the sound set happened to be pitched (e.g. steel drums). Adopting approaches similar to those suggested by Stevens [2], small groups of players were arranged where one player would be encouraged to create a musical idea that could be continued over some time using one of the synchronised instruments. The rest of the small group would be encouraged to listen for some time and to then take the opportunity to introduce a musical idea of their own. Using this concept, a simple structure for improvisation could be achieved where layers would gradually be added and then gradually stripped away using different combinations of instruments, players, patterns and sounds.

Other than further supporting the observations from the previous workshop in terms of ease of access and generative potential, comments were also offered with specific regard to the synchronised nature of the musical system. Most significant within this was a general reassurance that the various players did maintain a sense of musical control whilst interacting with the elements that were to some extent generative and/or rule-based. By way of example, one member of the group commented on the synchronised nature "how all the different instruments could link with each other, I enjoyed that". When asked whether he still had a sense of control within this, he confirmed that he did. Additional comments were made in regard to the synchronised timing being helpful in sustaining a sense of rhythmic 'togetherness'. One of the more able musicians within the group commented on this aspect in conjunction with a feeling of control "yes, it's just finding the right beats - at first I was pressing anything - then I was listening to where the beast were - then trying to remember them to get a rhythm".

\section{Discussion and Design Conclusions}

The overall concept of coordinating and synchronising a number of contrasting music-technologies has been shown to have considerable potential for assisting with group based improvisational activities. A combination of observation and individual comments suggests that this kind of approach could be highly enabling whilst still offering the individual players meaningful sense of control and therefore 'ownership'. Personal and professional experience also suggests that, though the general model of working with predefined musical patterns and textures in the ways described earlier can produce meaningful and rewarding results, the overall concept could be further enhanced. At a fundamental level, this might be achieved by facilitating composition within the system such that new rhythmic, harmonic and melodic patterns can be introduced by the user-composer. During the workshops, it was convenient to 
coordinate the various musical streams to share tonal and harmonic relationships. This was not the only compositional choice as it was also decided to not specifically follow a particular harmonic progression, instead allowing the harmonies to emerge organically within the chosen key. As these are compositional choices or constraints that will ultimately affect the nature of the improvisational experience it would be desirable to hand this aspect over to the control of the group. With this in mind, it could be both creative and empowering to implement a system that allows for compositional rules and constraints to be created or applied centrally in contrast to being reliant on those that are contained within specific instruments. This will create a central music-engine unit that is responsible for the generation and manipulation of an expandable library of musical ideas and patterns, effectively providing multiple (synchronised) instruments within a single unit.

The means by which any individual will engage with the system can be designed around the specific needs of that individual. An example was given earlier of an individual who expressed their pleasure over engaging with particular sounds but observation showed that the interaction required was not always easy. In contrast, there were other users for whom this mode of interaction was most effective. If the types of patterns and sounds that are produced by this type of technology are divorced from any specific interface and brought under control of the central music-engine, then specific and adaptable interfaces can be designed to allow any given individual to engage with the system. As suggested earlier, the concept of performancebehaviours for digital instrument perhaps needs to accommodate individual needs more than it currently does. With this in mind, the user-performer should perhaps be able to adjust the level of synchronisation to suit their physical and/or coordination challenges such that the timing assistance achieved still allows the performance experience to be meaningful. A prototype is now being developed that will bring together these various desirable design considerations into a working system. Though this is likely to be relatively basic in terms of its initial functionality, the aim will be to apply the system within an iterative series of workshops and redesigns, introducing additional functionality in a relatively organic way.

\section{References}

1. Bailey, D.: Improvisation: Its Nature and Practice in Music (2nd Edn), pp. 83-5, 140-2, British Library National Sound Archive, London (1992)

2. Stevens, J.: Search and Reflect, pp. 1-2, Rockschool, UK (1986)

3. Smith, R.: Teaching and Learning Free Musical Improvisation in UK and Norway. Improvisation Continuums Conference (2007)

4. Challis, B. P.: and Smith, R. Inclusive Technology and Community Music. (2008). In Proceedings of Accessible Design in the Digital World 2008 (2008)

5. Challis, B. P.: Octonic: an accessible electronic musical instrument. Digital Creativity 22 (1) pp. 1-12, Routledge, UK (2011)

6. Healey, R.: New technologies in music making. In: Community music: A Handbook, eds. Moser, P and McKay, G., pp. 161-179, Russell House Publishing Ltd (2005)

7. Malloch, J., Birnbaum, D., Sinyor, E. and Wanderley, M.: Towards a New Conceptual Framework for Digital Musical Instruments. In: Proceedings of 9th International Conference on Digital Audio Effects pp. 49-52 (2006) 